\title{
Rhétorique du discours contre la superstition dans les "Pensées diverses sur la comète" de Pierre Bayle: astronomie et littératureau service de la raison
}

\section{Alexandra Willaume Albertini}

\section{(2) OpenEdition}

\section{Journals}

Édition électronique

URL : http://journals.openedition.org/studifrancesi/1031

DOI : 10.4000/studifrancesi. 1031

ISSN : 2421-5856

Éditeur

Rosenberg \& Sellier

Édition imprimée

Date de publication : 1 novembre 2014

Pagination : 455-466

ISSN : 0039-2944

Référence électronique

Alexandra Willaume Albertini, «Rhétorique du discours contre la superstition dans les "Pensées diverses sur la comète" de Pierre Bayle: astronomie et littératureau service de la raison », Studi Francesi [En ligne], 174 (LVIII | III) | 2014, mis en ligne le 01 novembre 2014, consulté le 18 septembre 2020. URL : http://journals.openedition.org/studifrancesi/1031 ; DOI : https://doi.org/10.4000/ studifrancesi.1031 


\title{
Rhétorique du discours contre la superstition dans les "Pensées diverses sur la comète" de Pierre Bayle: astronomie et littérature au service de la raison
}

\begin{abstract}
In the historical context between the end of the Renaissance and the beginning of the Enlightenment the progress of the astronomic sciences is considerable, thanks to the development of the technical instruments used to observe the stars. The corollary is a resurgence of irrationalism through superstition, which becomes a way to understand the new cosmology when its global view stumbles between the Bible's vision and heliocentrism.

Pierre Bayle is a rationalist thinker who uses the critique of superstition about comets to promote the new knowledge with his Pensées diverses sur la comète (1683), though continuing to legitimate Christian faith.

The interdisciplinary character of this work can be examined starting from the analysis of its construction, and its epistemological and religious context. Bayle's method appears typical of those rationalist thinkers who are neither dogmatic nor atheist - in the broad sense of that time - nor extremely sceptical, and are aware of the new relationship between religion and science.
\end{abstract}

L'intérêt d'étudier la rhétorique du discours contre la superstition dans Les pensées diverses sur la comète de Pierre Bayle, réside dans le fait de montrer comment astronomie et littérature se mettent au service de la raison, et comment ensuite la raison est articulée chez Bayle au Christianisme, en dépit de l'échec du rationalisme chrétien, objet de nombreuses controverses à l'heure actuelle. L'essentiel de la démonstration physique se trouve au début de l'œuvre. Bayle y analyse la superstition des comètes, après le passage de celle du 26 décembre 1680 dans le ciel de Paris. Il reprend ainsi en 1682 dans La lettre sur la comète, et en 1683 dans sa réédition sous le titre des Pensées diverses ${ }^{1}$ la lutte anti astrologique qui anime de nombreux érudits depuis la fin de la Renaissance. Entre temps les sciences astronomiques se sont considérablement développées grâce aux progrès des outils techniques au service de l'observation des astres. Notamment la mise au point et l'augmentation de la puissance de la lunette astronomique, dont Galilée tirera en 1609 les thèses qui lui vaudront la condamnation de l'Église, dans son Dialogo sopra $i$ due massimi sistemi del mondo (1632), et ensuite le télescope de Newton (1668-1671). L'héliocentrisme entre de plus en plus en conflit avec la vision biblique du livre de la nature ${ }^{2}$. Cette crise de la pensée a pour corollaire une résurgence de l'irrationalisme, manifestée à travers l'hermétisme ou la superstition, comme moyens de décoder le monde et d'agir sur la nature, quand la vision de celle-ci vacille. En effet la superstition des comètes établit littéralement un rapport de cause à effet, ou à défaut de signe à effet entre des

(1) Nous utiliserons désormais l'abréviation PD.

(2) COPERNIC, De revolutionibus (1543), confirmé en 1610 par Galilée, mais système d'orbitation de la terre reconnu seulement au milieu du XVIII ${ }^{\mathrm{e}}$ siècle, ainsi que mise à l'index de l'héliocentrisme levée.
Galilée: «le soleil est plus probablement au centre de l'univers que la terre» (rq 55), Dialogue sur les deux grands systèmes du monde, Trad. R. Fréreux avec F. de Grandt, («Coll. Point sciences»), Paris, Seuil, 1992. 
éléments qui concrètement ou logiquement n'en ont aucun, en postulant une mauvaise influence de la comète sur la terre.

La lutte anti astrologique à la fin de la Renaissance a largement été étudiée par les chercheurs italiens Cesare Vasoli, Eugenio Garin ou en Angleterre par Frances Yates. Mais dans le courant du XVII ${ }^{\mathrm{e}}$ le problème se pose différemment, eu égard aux progrès du savoir dans ce domaine, auquel un libertinage savant va parfaitement adhérer. Cette pensée rationaliste envahit alors la littérature pour dénoncer les excès du merveilleux chrétien comme une forme de superstition qui ramène in fine la religion à ce type de croyance. Il est alors fréquent de voir ces auteurs (Vanini, La Motte le Vayer...) classés dans des catégories suspectes ou indiscutablement hérétiques, car refusant l'orthodoxie religieuse. Cet héritage libertin posera problème pour les penseurs comme Bayle qui leur succèderont dans la réflexion philosophique à la fin du siècle, et qui voudront intégrer les nouveaux savoirs, sans pour autant rejeter le sentiment religieux, mais seront taxés pour autant de libertins ou déistes, voire d'athées ${ }^{3}$ au sens extensif de l'époque. Exilé en Hollande depuis 1680, le penseur protestant est ainsi en butte à la censure en France.

La forme de «pensées diverses» assez ambigüe, masque en fait une construction rhétorique rigoureuse, élaborée pour souligner l'enjeu scientifique, mais aussi la portée religieuse du problème. Appelée par lui-même «petite dissertation» dans une lettre à son ami Minutoli (30 Mars 1683) ${ }^{4}$, et greffée sur la première forme épistolaire artificielle de la Lettre sur la comète, sa réflexion sera confirmée par de nombreux articles de son Dictionnaire historique et critique en 1695. Les deux œuvres font de leur auteur un exemple significatif d'une vision du monde à la fois chrétienne et savante par le truchement d'une littérature engagée, sans pour autant relever d'une vulgarisation scientifique simple.

Dans le domaine de l'astronomie, la construction du savoir a été pendant des siècles fondée sur une observation aléatoire des étoiles et sur des interprétations reposant sur la tradition, les mythes, l'intuition ou la conviction religieuse, nourrissant de proche en proche un chemin heuristique pour le moins ésotérique, et se confondant avec l'astrologie. Il nous paraît pertinent de montrer que Bayle opère une sorte de déconstruction de ce savoir à travers une critique réorganisée de la superstition des comètes.

On peut ainsi interroger l'interdisciplinarité des PD à partir de l'analyse du discours, en le situant dans son contexte épistémologique et religieux. On considèrera alors sa méthode comme représentative de l'engagement de penseurs rationalistes, qui ne sont ni dogmatiques, ni athées, et qui nuancent tout scepticisme de type exclusivement pyrrhonien. Tenant compte des nouveaux rapports entre science et religion, ils sont tournés vers la modernité sans être des adversaires de l'Antiquité, dans des genres qui s'affichent tout autant littéraires que philosophiques.

Notre propos envisage tout d'abord d'examiner la position dissidente de Bayle à l'égard de la posture calviniste en ce qui concerne la superstition astrologique. Nous interpréterons ensuite la spécificité de sa démarche sous l'angle rhétorique et scienti-

(3) Bayle est accusé d'être un athée par Jurieu par exemple, et même impie pour ses idées sur l'athéisme vertueux, ou d'être un sceptique pour sa méthodologie du doute quand il n'y a pas de preuve concrète. «Jurieu traite Bayle d'impie, de profane, d'homme sans honneur et sans Religion, de traître, de fourbe et d'ennemi de l'Etat, digne d'être détesté et puni corporellement», A. Prat, introduction de notre édition, pp. XX-XXI.
(4) A. McKenna et F. Vial-Bonacci, direction de l'édition numérique en cours de La Correspondance de Pierre Bayle, baylecorrespondence.univ -st-etienne.fr.

(5) P. BAYLE, Dictionnaire Historique et Critique, 4 voll., Genève, Slatkine, 1995. Nous utiliserons désormais l'abréviation DHC. 
fique conjugué, pour montrer cette nouvelle identité rationaliste fidéiste qu'il incarne, donnant une fonction théologique à la raison.

Le désir de lire les étoiles, né au moins 5000 ans avant notre ère, dans l'idée qu'une correspondance existe entre le ciel et la terre, est considéré comme une véritable science. En témoignent des relevés astronomiques en Mésopotamie. L'analyse du mouvement des astres par les mages chaldéens établit la première véritable astrologie/astronomie, et les mathématiques sont inventées dans le but de ces calculs, puisque «matematicci» signifie entre autres, «astrologues». Galilée ne fait donc que perfectionner ces calculs avec sa lunette au début du XVII siècle, disant en 1623: «il mondo è scritto in lingua matematica, e i caratteri son triangoli, archi ed altri figure geometriche» (Il saggiatore, Chap. VI) .

Ainsi dans un premier temps astrologie et astronomie sont encore largement confondues au $\mathrm{XVII}^{\mathrm{e}}$ siècle, malgré la perception qu'on en a aujourd'hui'. On discrimine dans l'astrologie de l'époque, astrologie prédictive ou judiciaire (relative au discernement, au jugement, du latin astronomia judiciorum ou astrologia judiciaria) de ce que Calvin appelait la «vraye astrologie» ou «astrologie naturelle», c'est-à-dire «l'ordre naturel et disposition que Dieu a mis aux estoilles et planetes» dans son traité Contre l'astrologie qu'on appelle judiciaire et autres curiositez qui regnent aujourd'bui au monde $(1549)^{8}$. Il y tolère la possibilité de prodiges divins derrière certaines apparitions de comètes, mais il condamne leur superstition en général comme diabolique en s'appuyant sur les nombreuses interdictions bibliques liées aux divinations'. Par ailleurs l'astrologie agricole est reconnue par lui. Mais d'après Calvin l'appellation de «mathématiciens» pour désigner les astrologues, est souvent un «manteau» pour cacher des hérésies dites «curiosités» au sens latin des curiositates, c'est-à-dire superstitions païennes ${ }^{10}$. Bayle s'affiche calviniste, et cette influence a été largement prouvée (il l'affirme d'ailleurs dans le DHC à plusieurs reprises). Il est partisan de cette lutte, mais il va pousser plus radicalement le point de vue de Calvin, en ne tolérant aucune restriction d'ordre prodigieux. Le DHC le confirme avec le portrait élogieux d'Anaxagore, philosophe du $\mathrm{V}^{\mathrm{e}}$ siècle avant J.-C., qui affirme que les astres ne sont qu'une masse incandescente même si tout le monde y voit la manifestation des Dieux. Accusé d'athéisme et condamné à mort, le penseur grec incarne la première vision rationnelle des comètes qu'il considère comme des éléments naturels (DHC, vol. I, p. 215). À cette époque de l'Antiquité, envisagée par le psychiatre allemand Karl Jasper comme un âge axial de l'humanité car l'homme pense pour la première fois le sens de son destin personnel grâce à la philosophie ${ }^{11}$, on constate que la discussion

(6) Et cela replace l'homme au cœur de ses préoccupations les plus profondes au sujet du questionnement existentiel. La véritable astronomie au sens où nous l'entendons à notre époque coïnciderait avec la découverte des satellites de Jupiter le 7 janvier 1610, même si les hollandais en posent déjà l'hypothèse quelques années auparavant. Enfin la création de l'observatoire de paris en 1667 entérine la perspective véritablement scientifique de l'astronomie. Voir J. P. VERDET, Une histoire de l'astronomie, («Coll. Point Sciences»), Paris, Seuil, 1990.

(7) En témoignent les circonstances de l'apparition de la comète qui intéresse Bayle le 26 décembre 1680, à 17 h dans le ciel de Paris. La société érudite en rit, les parisiens sont dans l'effroi et le gouvernement de Louis XIV craint un ravivement de l'Affaire des poisons.

Par ailleurs les analyses de la correspondance des disciples de Galilée au XVII ${ }^{\mathrm{e}}$ siècle par E. GARIN le montrent clairement: ceux-ci déplorent le peu d'intérêt du public pour les sciences mathématiques, alors que l'astrologie populaire reste très en vogue. Mais celle-ci est condamnée par l'Eglise. Le zodiaque de la vie (Polémiques antiastrologiques à la renaissance), Paris, Les belles lettres, 1991, p. 24.

(8) Edition critique O. Millet, Genève, Droz, Textes littéraires français, 1985, pp. 55-53 (Les caractères italiques sont dus à l'auteur).

(9) Quand Yahvé, par exemple, dicte les tables de la loi à Moïse, et lui dit «vous ne pratiquerez ni divination, ni incantations» (Le Lévitbique, XIX, 26). Commandement que l'on retrouve décliné sous plusieurs formes dans l'ensemble de la Bible.

(10) «Superstitions païennes» selon le Dictionnaire latin-français des auteurs chrétiens par A. BLAISE, Turnhout, Brepols, 1954-1967.

(11) Origine et sens de l'bistoire (Vom Ursprung und Ziel der Geschichte), Paris, Plon, 1954. 
astronomique est déjà posée telle qu'on la retrouvera à l'aube des progrès technologiques, physiques et mathématiques de la fin du XvI siècle. Mais l'ambigüité astronomie/astrologie ne permet pas de séparer la dimension cosmologique de l'astronomie de sa dimension interprétative, bien que Ptolémée l'ait lui-même tenté en son temps dans le Tétrabiblos. L'homme cherchera toujours à décoder dans le ciel, les signes divins qui lui donneront des indications sur son avenir pour le rassurer sur le sens de sa vie sur terre en dépit de la mort.

Ainsi Bayle, bien que considérant dans les Pensées diverses les oracles antiques comme diaboliques selon la thèse chrétienne, aussi bien catholique que protestante, (titre du chapitre CXI «les démons entretenoient les superstitions en produisant des prodiges...», qui démontre sans ambigüité la présence satanique derrière l'oracle de Delphes), se refuse à laisser aux comètes la moindre possibilité divine ou diabolique, et rompt de ce fait avec la tradition théologique de sa propre religion.

Qu'est ce qui le motive pour prendre de telles libertés?

Ce qui pousse Bayle à investir le sujet assez longuement se déplace vers un autre intérêt, sans compter le DHC où l'article Nigidus Figulus Publius confirme catégoriquement sa position anti astrologique: «il seroit absurde de dire que la connoissance de la vertu des étoiles peut révéler l'avenir» (vol. III, p. 509, Note E). Pour lui il est indigne de la sagesse divine de transformer de vulgaires astrologues en prophètes, par le biais du «travail ridicule de dresser un horoscope». La prédiction entrant par ailleurs en contradiction avec la liberté humaine d'agir, sans compter son interdiction biblique.

Dans les PD, Bayle aborde le sujet en partant de l'Antiquité pour arriver à la France de son époque du chapitre XVII au chapitre XXII en déniant l'influence des comètes radicalement dans le titre du chapitre XVII «que l'astrologie, qui est le fondement des prédictions particulières des comètes, est la chose du monde la plus ridicule» (I, p. 56). On retrouve le terme «ridicule», comme cela sera le cas dans le DHC. Il compare ainsi les prédictions des astrologues à celles des poètes pour en souligner le caractère fictif, les nommant «extravagances» (I, p. 63). Il trouve même impertinent qu'on croit à leur influence, et «refuse de (s')amuser à prouver ce qu'(il) avance si fièrement contre la vanité de l'astrologie judiciaire» (I, p. 65). Comme il le dira encore dans l'article «Morin jean Baptiste»: «Les plus grands hommes d'état se laissent infatuer de l'astrologie judiciaire, et que même dans le XVII ${ }^{\text {èe }}$ siècle, on n'a pas été exempt de cette folie à la cour des plus grands princes d'Europe» (DHC, vol. III, p. 426).

«Fausseté de cet art chimérique» (PD, I, p. 65), «Absurde» (p. 66), «Impostures» (I, p. 67), «Imagination» (I, p. 73) sont des termes catégoriques mais l'auteur reconnaît que la faiblesse fait céder à la croyance (I, p. 69). Un même champ lexical est utilisé par Calvin, avec «folles opinions», «fantaisies extravagantes», «fausses et méchantes opinions», «folie» ${ }^{12}$. Dans le DHC, le portrait de Carnéade, philosophe grec, lui permet dans une note, de développer l'idée que l'avenir ne peut se prédire à travers l'exemple des oracles (vol. II, p. 64 et suivantes, note L). Il dit en effet que «sa dispute contre les oracles d'Apollon avait quelque force».

Les choses futures ne peuvent être prédites que si elles dépendent d'une cause nécessaire. Si celle-ci est ignorée, comme d'autres causes efficientes, on ne peut rien savoir. Donc le futur est en relation avec le passé. Le problème étant accusé par le libre arbitre humain, imprévisible. Bayle associe ces réflexions sur Carnéade à la morale chrétienne, et s'étonne qu'on ait laissé le philosophe débattre sur la question 
car ses «raisons étaient fort propres à ruiner de fond en comble toutes les divinités païennes». Selon Benvéniste, le sens étymologique de la superstition était en effet à l'origine «divination du passé» ${ }^{13}$.

Le paganisme antique, la nature humaine crédule et les religions infidèles actuelles sont, d'après Bayle, des explications au crédit permanent de l'astrologie, en tant que postures fondées sur l'erreur et la superstition, cautionnées par le plus grand nombre (PD, I, p. 80). Bayle constate cependant la croyance relative de grands hommes dans l'astrologie, comme Albert le Grand. Mais l'auteur souligne leur excès quand ceux-ci ont eu la prétention de faire l'horoscope de Jésus Christ (PD, I, p. 76), comme cela a été aussi l'«audace» de Cardan (DHC, vol. II, p. 54). C'est pour Albert le Grand une «témérité», puisque «les vertus et miracles» du Christ sont «d'un ordre tout à fait surnaturel» et ne reposent pas sur un agencement prometteur d'étoiles à sa naissance (PD, I, p. 76). À l'arrivée c'est la foi dans la révélation biblique qui dicte le point de vue de Bayle. Elle est affirmée tout au long de son œuvre, et illustrée dès les premières lignes de «l'éclaircissement sur les Pyrrhoniens»:

J'établis d'abord comme la base de ce troisième Ecclaircissement cette Maxime certaine \& incontestable, que Le Christianisme est d'un ordre surnaturel, son analyse est l'Autorité suprême de Dieu nous proposant ses Mystères, non pas afin que nous les comprenions, mais afin que nous les croyions avec toute l'humilité qui est due à l'Etre infini, qui ne peut ni tromper, ni être trompé. C'est là l'étoile polaire de toutes les Discussions, \& de toutes les Disputes, sur les articles de la Religion que Dieu nous a révélés par Jésus-Christ [...] Il faut nécessairement opter entre la philosophie et l'Evangile ${ }^{14}$.

L'autorité de l'astrologie n'est fondée selon Bayle que sur le plus grand nombre (I, p. 80), ce qui est source d'erreur, comme le dira aussi Fontenelle avec sa célèbre métaphore du «torrent» (Histoire des oracles, 1687), que reprend Bayle dans l'article «Epicure» du DHC, en parlant des préjugés de l'histoire: «Plusieurs grands hommes entraînez par le torrent, ont suivi de siècle en siècle les préjugez établis, sans examiner les choses au fond» (vol. II, p. 371, note N).

Soulignons maintenant la démarche scientifique très particulière des PD à travers sa rhétorique du discours.

En effet la démonstration de Bayle pour prouver que les comètes ne sont ni le signe ni la cause d'un évènement sur terre, relève d'une approche de logique quasi mathématique. Celle-ci détermine rationnellement le «cause à effet» de sa pensée pour contrer justement cette absence de cause à effet qui caractérise la superstition, à travers une démonstration des objets physiques. «Quoi que je ne sois pas astronome, ni d'effect ni de profession, dit-il, je ne laisse pas d'étudier soigneusement tout ce que les plus habiles ont publié sur cette matière» (PD, I, pp. 25-26). La remise en cause de la théorie de l'influence des astres est l'objet de la «Première partie» de l'œuvre. Pour commencer l'auteur examine les aspects concrets dans la position des comètes par rapport à la terre. Les lunettes télescopiques, et les calculs mathématiques (la garantie des mathématiques sera posée dans l'article «Zénon l'épicurien» du DHC: «les mathématiques sont la science la plus évidente et la plus certaine dans les connoissances humaines» (vol. IV, p. 547, note D) montrent que celles-ci en sont très éloignées, et n'impliquent donc pas de cause efficiente directe: «il est fort probable, dit-il, qu'elles n'ont pas la vertu de produire quelque chose sur la terre» (PD, I, p. 41). Mais dans

(13) E. BENvÉNISTE, Le vocabulaire des institutions indo-européennes, Paris, Ed. de Minuit, 1969, 
un souci de raisonner avec exhaustivité et objectivité sur le sujet, Bayle pose l'hypothèse dans la page suivante, d'un envoi de matière possible depuis ces astres. Il en déduit tout naturellement un affaiblissement du pouvoir de cette matière itinérante, et donc de son influence au moment d'un impact potentiel sur notre planète, à cause du long voyage opéré, puisque les télescopes attestent d'une longue distance entre les comètes et nous. Elles deviendraient éventuellement une sorte d'«exhalaison» inopérante (Chap. XIII). Il ajoute comme preuve que nous ne recevons d'elles que la lumière que nous voyons, par réflexion du soleil, et que celle-ci ne bouleverse pas la matière (I, p. 43). Newton prouvera en effet dans les Principia en 1687 que la lumière scintillante de la queue de la comète est de la vapeur exhalée à cause de la chaleur liée à la promiscuité du soleil ${ }^{14}$. Cela est renforcé chez Bayle par un constat statistique qui atteste dans l'Histoire de l'existence d'épisodes malheureux en dehors de la parution de comètes dans le ciel, et vice versa, exprimé par le chiasme: «Il y a des malheurs sans comètes et des comètes sans malheurs». (I, p. 125). De plus elles ne sont pas visibles au même moment depuis certains endroits de la terre, et pendant ce temps des bonheurs et des malheurs arrivent simultanément. La malédiction peut donc être relativisée. Pour ce faire il prend l'exemple des guerres dans plusieurs chapitres, calamité pour le perdant, la guerre est une réussite pour le vainqueur. Bayle étendra alors sa thèse à de nombreux phénomènes comme les éclipses.

Examinons maintenant l'état des savoirs par rapport à cette superstition.

La croyance dans le géocentrisme perdure au XVII malgré la thèse copernicienne $^{16}$. Elle pouvait donc soutenir momentanément la superstition dans l'influence des comètes sur la terre. Par ailleurs au moment où Bayle écrit la Lettre sur la comète, Newton n'a pas encore prouvé l'attraction terrestre, il le fera à partir de 1684 (publication dans les Principia en 1687). Ce qui évite un amalgame dangereux entre géocentrisme et attraction terrestre.

Bayle doit donc démêler deux choses qui se juxtaposent au moment de la rédaction des $\mathrm{PD}$, à propos des «corps flottants» comme on nommait les météorites à cette époque, les confondant souvent avec les comètes ${ }^{17}$. En homme éclairé, il a admis l'héliocentrisme (il cite Copernic dès 1678 dans sa correspondance' et rappelons qu'il suit scrupuleusement les publications scientifiques sur les progrès des savoirs puisque par ailleurs il dirige la revue «Nouvelles de la République des Lettres» fondée justement en 1683) et il peut donc déduire raisonnablement de ses connaissances le manque de pouvoir de la matière des comètes sur la terre. De plus les cartésiens vantaient l'inertie au détriment de l'attraction terrestre, c'est-à-dire la conservation de la force immuable de l'objet en mouvement rectiligne, même en cas de choc avec un autre objet dont la cause a été produite par Dieu (postulat). C'est-à-dire présupposent un système qui donne au mouvement une trajectoire rectiligne que Newton étudie à partir de 1679 (d'après les Principes de la philosophie de Descartes qui serviront de base aux travaux de Newton, même si ce dernier le réfutera).

On peut émettre ici deux hypothèses: soit Bayle commet l'erreur des cartésiens, et selon «les premiers principes du bon sens», au chapitre XXII, il établit ainsi le manque de rapport de cause à effet entre la comète et des évènements terrestres. Il

(14) Texte appartenant à la deuxième édition du DHC, troisième éclaircissement (1702), suite à la censure du consistoire de Rotterdam du 20 décembre 1698, vol. IV, p. 641 (Les caractères italiques sont dus à l'auteur).

(15) Newton, Principia, principes de mathématiques de la philosophie naturelle, Préface de Vol- taire, Paris, Dunod, 2005, Livre III, proposition 11, p. 393.

(16) Perspective de l'astrologie hellénistique, qui envisage l'univers dans sa finitude, et qui croisera la cosmologie religieuse fondée sur les Écritures.

(17) J. P. VerDET, op. cit., p. 192. 
montre en effet dans le chapitre CVIV que les comètes tournent autour de la terre (argument rationnel fondé sur l'observation) et ne visent aucun peuple en particulier, ce qui revient à postuler une loi de permanence dans le mouvement, comme Descartes (selon le postulat de la conservation de la force en mouvement rectiligne). Soit Bayle anticipe par ses observations philosophiques, sur la loi de gravitation de Newton, qui établira que les corps célestes échappent à l'attraction terrestre grâce à leur vitesse qui les place en une sorte de satellisation en orbite elliptique allongée (livre III proposition 11, à propos justement de la comète de décembre 1680). D'ailleurs les travaux de Halley sur la comète de 1682 viennent de montrer l'orbite elliptique et le caractère prévisionnel des passages. De plus à cette époque Bayle est en rapport avec Huygens ${ }^{18}$ qui trouve absurde la gravitation universelle, mais qui travaille depuis 1673 sur l'attraction terrestre. Huygens rencontre Newton en 1681, mais Bayle ne mentionne pas Newton dans sa correspondance.

Synthétisant ces différents points de vue plus ou moins justes, et en doutant raisonnablement que Bayle ait suivi les cartésiens dont il commence alors à se distancier $^{19}$, on peut penser qu'il conjugue logique et science pour proposer une thèse valide, c'est à dire en fait les travaux de recherche de Newton jusqu'en 1687, même s'il apparaît qu'il en ignorait les éléments purement scientifiques au moment des PD.

Il ne reste plus à Bayle qu'à examiner la possibilité du signe, en s'interrogeant sur un pouvoir possible des comètes dans ce sens. Or il y a déjà doute sur la question, puisqu'il n'y a pas de preuve de signe à effet si ce n'est la coïncidence très aléatoire de malheurs au moment du passage des comètes (critère déjà analysé dans sa démonstration précédente sous la forme de «preuves» et de «raisons»). De plus Dieu ne laisserait pas selon lui, une telle ambiguitté en suspend, qui suggèrerait une superstition interdite. (L'objet est ici au cœur du débat à propos des causes occasionnelles, et du conflit entre évolutionnistes et catastrophistes, davantage idéologique que scientifique, ce qui nous fait comprendre le problème de superstition qui en est en cause $)^{20}$. On retombera alors sur les croyances du consensus, et le problème de leur tradition, dans la «Deuxième partie», où l'auteur invite le lecteur à se méfier des faux miracles qui nuisent au Christianisme (Tome II, chap. CCXVII, p. 236). Il ponctue ainsi le premier aspect de sa thèse «ce serait abuser de la patience d'un habile homme que de lui expliquer ceci plus au long» (PD, I, p. 147).

La grande circonspection et la rigueur dont fait preuve Bayle à propos de l'astrologie se retrouvent dans l'article «Kepler» du DHC à propos d'astronomie. Même s'il considère le savant comme «un des plus grands astronomes de son siècle» (vol. III, p. 2), Kepler (chargé d'études par Tycho Brahé) établit dans son Astronomia nova (1609) trois lois sur le mouvement des planètes, dont la troisième inspirera les calculs de Newton. Bayle ajoute que ses théories restent suspectes car il croyait que la terre a une âme, des pensées et des sentiments alors que rien ne peut nous le prouver. En particulier, Bayle cite la croyance de l'astronome dans le fait que la pluie est la suée de frayeur de la terre quand elle se sent menacée par une comète (vol. III, p. 3, note D). On comprend

(18) Il en suit les travaux (Lettre à Henri Justel le 24 juin 1684 à propos des lunettes sans tuyau), et adresse à Huygens la seconde édition de La lettre sur la comète, c.a.d les PD, car il le trouve «universel», même s'il précise que lui-même ne parle pas de physique, de géométrie ou d'astronomie (Petit mot accompagnant l'envoi de l'ouvrage le 14 septembre 1683).

(19) Bayle commence à prendre ses distances avec le cartésianisme en 1681, comme en atteste une lettre à son frère Jacob (29 mai): «hypothèse in- génieuse qui peut servir à expliquer certains effets naturels mais au reste j'en suis si peu entêté que je risquerois pas la moindre chose pour soutenir que la nature se reigle ou se gouverne selon ces principes là». Il le réfutera plus tard intégralement, en 1693 dans une lettre à Huygens et dans son article «Spinoza» du DHC.

(20) J. P. Verdet, op. cit., p. 297, dans le questionnement humain à propos de l'intervention possible de la providence ou du hasard. 
le jugement de Bayle sur ce qu'il doit considérer comme une superstition... Par ailleurs dans le DHC, il dénie les facultés des astrologues à de nombreuses reprises, donnant purement et simplement des exemples de diverses erreurs, sans commentaires, comme l'article «Nicolas Peiresc» qui s'achève sur la phrase: «Les astrologues avoient prédit qu'il auroit femme et enfans, et néanmoins il ne fut jamais marié» (vol. III, p. 639). Bayle.

Examinons maintenant les conséquences à propos du lien à la religion chez

La thèse de Bayle, sur le fond, n'est pas pour autant novatrice. Dans son ouvrage I miti e gli astri, Cesare Vasoli établit la tradition des détracteurs de la superstition des comètes à la fin du XVI ${ }^{\mathrm{e}}$ siècle ${ }^{21}$. La polémique se rattache à l'origine au fait que derrière les croyances astronomiques se cache la figure du démon (du démonique au démoniaque): «nelle credenze astrologiche sono ben presenti ed operanti i residui di una «religio daemonica», fondata sull'identificazione dei «principi» astrali con potenze e forze diaboliche» selon l'auteur Bruto ${ }^{22}$.

La superstition des comètes implique ainsi à cette époque la peur permanente $\mathrm{du}$ diabolique. La comète se rapproche sans doute dans l'imaginaire collectif de la vision eschatologique de «l'étoile incandescente» tombant du ciel dans l'Apocalypse de Saint Jean (VIII, 10-11), ou «grand astre flambant comme une torche» suivant les traductions bibliques. Avec l'image de «sa queue qui balaie le tiers des étoiles (les messagers de dieux déchus) et les précipite sur terre» (Ap. J. XII, 4). Par ailleurs on croyait à des charriots de feu qui transportaient les âmes, comme celle du prophète Elie, sauvée par Dieu, sur qui Bayle jette le discrédit dans le DHC, évoquant les «contes» qui le concernent (vol. II, p. 347).

La querelle des comètes, que l'auteur italien analyse entre 1578 et 1580, ressemble fort alors à celle de 1680, opposant rationalistes et superstitieux, et même une frange de penseurs qui envisagent la compatibilité de l'astrologie mathématique et divinatoire. André Dudith y démontre déjà rationnellement au XVI siècle l'absurdité de leur superstition en prenant appui, semble t-il comme Bayle, sur la réalité dans son Commentariolus (1579) ${ }^{23}$. Son dessein est de limiter la superstition pour favoriser la connaissance de Dieu, et de ramener la croyance à un examen scientifique qui écarte ces postulations irrationnelles. Selon lui elles suscitent la bile qui déclenche les guerres dont on pense que la comète peut être le signe ${ }^{24}$.

Ainsi Bayle ne serait pas le premier, mais il est celui qui entérine la démonstration à l'aube du siècle des lumières, à l'aide des nouveaux savoirs, et d'une méthodologie rigoureuse, et somme toute comparatiste ${ }^{25}$. En particulier parce que la polémique de la fin du XVI e siècle dérive naturellement sur la question des causes, et donc la querelle des causes occasionnelles, dont elle met en place les débats, auxquels Bayle, un siècle plus tard participera avec le regard éclairé par les progrès des sciences.

Les nouveaux savoirs du XVII ${ }^{\text {e }}$ siècle ont donc contribué sans doute à relancer la question qui ne trouvait pas de consensus à la fin du XVI ${ }^{\mathrm{e}}$ siècle, et ont montré la nécessité d'une méthode scientifique. L'approche humaniste érudite de la superstition, antérieure aux progrès purement technologiques du télescope, a jeté des bases que Bayle peut reprendre au service de son sujet. Il confirme ainsi rétrospectivement les postulations des savants de la fin du XVI ${ }^{\mathrm{e}}$ siècle.

(21) C. VAsoli, I miti e gli astri, Napoli, Guida editori, 1977, pp. 362-387.

(22) Ibid., p. 365, «Dans les croyances astrologiques sont bien présentes et opérantes les reliquats d'une religio daemonica fondée sur l'identification des principes des astres au pouvoir et à la force diaboliques» (Trad. A.W.A).
(23) Ibid., pp. 366-367

(24) Ibid., p. 368.

(25) Objet de ma conférence au congrès international de l'AILC (Paris Sorbonne, juillet 2013): «Savoir astronomique et postulations philosophiques dans la thèse de Bayle sur la comète», qui est la suite de cette réflexion. 
Mais ces éclaircissements ne révèlent pas d'après nous une vulgarisation scientifique au premier degré. Bayle poursuit un autre but. La discrimination des superstitions parait nécessaire au renforcement du véritable irrationnel religieux, relevant du merveilleux chrétien. La validité des miracles et des prophéties bibliques auxquelles Bayle adhère fermement, ne peut qu'être confortée par la mise à l'écart de la superstition. En particulier celle du «porte malheur» dans ces liens au diabolique. Le combat est ici davantage d'ordre parénétique que scientifique pour le chrétien fervent qu'est Bayle. Il faut de plus entériner la limitation du pouvoir satanique en démystifiant les superstitions. Car ces croyances flattent selon l'auteur, le diable en tant que péché de divination, qui dérive souvent vers l'idolâtrie, autant condamnable par l'Eglise ${ }^{26}$. En contraste, le pouvoir de Dieu peut être réaffirmé.

Cependant les outils de la raison se heurtent ici à la foi, qui ne peut être prouvée, et qui ne le doit pas selon son principe intrinsèque, quand Bayle veut montrer la légitimation de l'irrationnel religieux en dépit de la condamnation de l'irrationalisme superstitieux.

Instrumentaliser une méthode scientifique rationnelle garantit la réussite d'un raisonnement fondé sur l'empirisme, aidé du progrès de la technologie et de l'art de la rhétorique, mais peut fragiliser la foi. D'autant plus quand l'auteur se détache d'une tradition religieuse liée à la théologie civile.

La différenciation des mathématiques et des sciences naturelles d'une part (selon la méthode qui sera celle de Galilée), et d'autre part les nouvelles méthodes philologico-humanistes dans le débat à propos des comètes, attestent selon Vasoli du passage vers le monde moderne, au-delà de la simple confrontation d'univers ${ }^{27}$. En effet, la mutation des mentalités et des pratiques fait en quelque sorte le lit de l'interdisciplinarité du savoir dont Bayle sera un exemple frappant. L'humanisme semble ici le fondement d'un intellectualisme en voie de diversité, préparant une nouvelle identité rationaliste de penseurs qui joignent leur culture des humanités à une approche empirique du monde, refusant de céder par ailleurs sur leur foi.

Il est donc nécessaire pour protéger le sentiment religieux, d'englober dans sa démarche rationnelle, le doute inhérent à la foi. Puisque de toute façon les nouveaux savoirs contribuent à sa remise en question. Nous dirons donc qu'il faut replacer le combat de Bayle contre la superstition dans la perspective d'une méthodologie scientifique appuyée sur la recherche de la vérité depuis la Renaissance, pour constater qu'en tant que réformé, Bayle est en quête d'un sens spirituel validé dans les Evangiles, et dans la réalité du monde en même temps.

Il incarne une tentative rationaliste de faire le lien entre la raison et la foi en ne rejetant pas les nouveaux savoirs, et en stigmatisant/démystifiant la superstition que leur crainte engendre. Cependant cette conciliation de la raison et de la foi reste illusoire si on les envisage sur le même plan.

La conciliation est restée avortée dans l'histoire de la pensée chrétienne. La «théologie naturelle» qui donne une fonction rationnelle à la religion chez Saint $\mathrm{Au}$ gustin au v viècle, est intégrée dans les thèses thomistes huit siècles plus tard, quand Saint Thomas évoque une «science du surnaturel» en accédant par ailleurs directement aux textes d'Aristote qui avait lui-même posé l'hypothèse d'une conciliation entre philosophie et religion, la seconde apportant des réponses à la première. Foi et raison étant deux créations divines elles paraissent tout naturellement conciliables au

(26) Voir l'article «superstition», qui établit l'historique du concept dans le Christianisme, in Dictionnaire de théologie catholique (qui contient l'exposé des doctrines de la théologie, leurs preuves et leur histoire). Sous la direction de A. VACANT E. Mangenot - E. Amann, Paris, Letouzey et Ané, t. XIV/2: Scholarios-Szczaniecki.

(27) C. VAsOLI, op. cit., p. 11 et p. 251. 
Moyen Âge. Mais la rupture avec l'Aristotélisme va entériner la négation de l'allégorie cosmique qui établit des correspondances entre le ciel et la terre, en privilégiant la conception d'un univers naturel, sur lequel la raison met du sens non à travers des signes, mais en s'appuyant sur l'analogie formelle. Le conflit se posera au fur et à mesure des progrès de la connaissance. Le besoin métaphysique entrant en contradiction avec l'analyse du monde et son évolution. Après l'école padouane qui a séparé foi et raison, le schisme anglican et la réforme, la raison est suspecte pour beaucoup d'érudits chrétiens au XvI ${ }^{\mathrm{e}}$ siècle. «Putain du diable» pour Luther, elle est un don naturel qui s'est corrompu pour Calvin.

En effet les débats astrologico-astronomiques dont nous parlons entre le $\mathrm{XVI}^{\mathrm{e}}$ et le XVII ${ }^{\mathrm{e}}$, certes éclairés par les nouveaux savoirs technologiques, physiques et mathématiques, montrent la nécessité d'une explication rationnelle du monde en même temps que la croyance dans la perception surnaturelle de l'action du divin pour les penseurs comme Bayle, qui ont la foi. Et la superstition doit être bannie à l'aide de la raison pour laisser sa place à la foi. Ce qui fait dire à Jean Boisset qu'on peut parler, en ce qui le concerne, d'«une sorte de probabilisme réfléchi fondé sur l'incertitude dans laquelle est enfermé l'esprit humain» ${ }^{28}$.

À notre époque ce fidéisme profond de Bayle a parfois été soupçonné d'être le paravent d'un certain athéisme, sous prétexte que sa prose ne contient pas d'emphase mystique $^{29}$. Au premier abord, la rigueur quasi mathématique de la démonstration dans les PD, nous montre il est vrai une écriture rationaliste, bridant toute spiritualité sous forme ostentatoire. Bayle revendique ses croyances simplement, sans glose. Il souhaite faire l'apologie de la vérité, en restant crédible et tolérant. Il cite les Écritures comme arguments d'autorité, plus que pour exalter un certain lyrisme religieux. Il est même capable de les remettre en question sur le plan formel:

Plus on raisonne sur les attributs de Dieu conformément aux notions les plus claires, les plus grandes, et les plus sublimes de la Métaphysique, plus on se trouve en opposition avec une foule de passages de l'Ecriture. Quoique cette opposition ne soit pas fondée sur les choses mêmes, mais sur la différence des styles, il est pourtant malaisé de la lever d'une manière qui satisfasse tous les esprits ${ }^{30}$.

Le but est de faire réfléchir, mais pas de faire un prosélytisme dogmatique qui a tant nuit à la religion dans ses dérives fanatiques.

La rigueur scientifique ne laisse place ensuite au second degré à l'hypothèse fidéiste que quand il n'y a plus de preuve logique ou empirique, et donc quand elle a atteint ses limites. Le doute qui prélude à cette pause envisage le fidéisme sans rejeter la raison comme inutile, puisqu'elle a été nécessaire jusque là. Il s'agit d'une «époché» de type logique et même chrono-logique dans l'approche du sentiment religieux.

Le scepticisme qui s'exprime alors dans ses écrits, revient davantage à une prise de conscience de l'erreur des dogmatismes humains, qu'à une posture systématique de type pyrrhonien. Dans son troisième «éclaircissement» de la publication du DHC, il montre la compatibilité du scepticisme avec la religion. Dans l'article «Pyrrhon» le chrétien et le sceptique sont rapprochés: le pyrrhonisme «peut avoir les usages pour obliger l'homme par le sentiment de ses ténèbres, à implorer le secours profondément sceptique» (vol. III, p. 733). Mais il semble qu'il le prenne dans son sens extensif. «Le

(28) J. BoISSET, «Pierre Bayle et l'enseignement de Calvin», in revue «Baroque», numéro VII, 1975, p. 13.
(29) E. Labrousse, Pierre Bayle: Hétérodoxie et rigorisme, La Haye, Martinus Nijhoff, 1964. p. 110. (30) «Avertissement au lecteur» de l'édition de 1699 des PD, t. I, p. 19. 
tort est toujours du côté de ceux qui affirment, que du côté de ceux qui suspendent le jugement» dit-il déjà dans les PD (I, p. 52). La sagesse sceptique inviterait donc le penseur à reconnaître, non pas l'impuissance de la raison comme dans le fidéisme radical, mais le nécessaire abandon de la raison à un certain moment...

Cette liberté de penser dont Bayle fait l'apologie, en dehors du jugement des hommes (auquel il ne veut obéir), et seulement soumise au divin, prouve selon Jacques Prévot son libertinage ${ }^{31}$. D'après ce dernier, les PD sont la preuve d'une pensée «à l'abri des instruments de mesure de la théologie et du dogmatisme» ${ }^{32}$. Certes, mais l'œuvre montre surtout un cheminement de pensée qui ne part pas de la remise en cause systématique du dogmatisme dans une posture libertine qui s'affranchit d'emblée de toute tradition, mais à l'inverse, qui aboutit d'après nous à un scepticisme sur la validité des dogmes dans leur forme ambigüe. La querelle actuelle sur la religiosité des libertins mérite ici d'être dépassée. Classer Bayle ou pas chez les libertins, paraît difficile si on se fonde sur les définitions problématiques et contradictoires qui circulent sur le libertinage érudit ${ }^{33}$. En examinant la méthode de réflexion de l'auteur on est sans doute plus à même de cerner son identité profonde.

Pour conclure, il semble donc raisonnable de dire que Bayle instrumentalise véritablement dans les $\mathrm{PD}$, la rhétorique et les sciences en tant que disciplines rationnelles, au service de la foi. Transposé, le doute progressif, méthodologique de Bayle, appuyé sur les nouveaux savoirs, participe du tâtonnement du croyant, à partir d'un constat d'échec de la religion institutionnalisée, complété par les progrès des sciences, mais qui ne remet pas en cause la foi chrétienne. Son scepticisme serait davantage à ce titre le produit du doute empirique, que le libertinage d'esprits érudits qui postulent finalement une forme personnelle de déisme, comme c'était le cas pour les libertins de la première moitié du siècle, dont nous pensons qu'il se démarque réellement. Il s'appuie en humaniste, sur la critique historique pour développer en toute liberté son propre scepticisme chrétien, rationaliste et moderne.

Soumis non pas à la religion chrétienne, mais à la théologie, comme il le souligne dans l'avis au lecteur de l'édition de 1682, utilisant «les mêmes armes de la piété et de la religion, desquelles on s'est servi jusqu'ici en faveur de ces présages» et «ne demandant autre juge que la Théologie» (I, p. 5). Par ailleurs le DHC réfute à plusieurs reprises l'esprit libertin sous le prétexte qu'il valorise trop la raison. Il le souligne par exemple dans l'article «Pyrrhon», en souscrivant à l'opinion de Pascal (vol. III, p. 733), il dénonce l'argument libertin de Dieu auteur du péché pour expliquer le mal dans l'article «Pauliciens» (vol. III, p. 631), ou bien dénie celui que Dieu fait des lois sur le bien, qu'il viole lui-même en damnant les hommes (vol. III, p. 633). Comment Bayle pourrait-il à ce titre être un vrai libertin, quand on prend nécessairement le terme dans le sens où lui-même devait l'entendre comme ses contemporains?

Bayle met selon nous en place les éléments d'un Christianisme moderne, tolérant et éclairé, qui intègre les progrès des sciences pour réfuter les superstitions afin de faire la part du véritable merveilleux chrétien. Le choix de la raison peut soutenir pour lui, à ce stade, ensemble la vérité de la science et la vérité de la foi. Mais sa démarche sceptique par ailleurs montre en fait qu'aux confins de la raison se trouve

(31) Libertins du XVII siècle, éd. J. PREVOT, Paris, Gallimard, «Bibliothèque de la Pléiade», vol. II, 2004, p. 1724.

(32) Ibid., p. 1725.

(33) Voir les colloques suivants:

- Clermont-Ferrand, Avril 1986, Le libertinage érudit dans la première moitié du XVII siècle, actes CERHAC.

- Bruxelles, Mai-juin 2011, De l'usage du terme «libertin»: invectives et controverses aux XVI et XVII siècles.

Voir aussi la bibliographie importante donnée sur le sujet par Jean Pierre Cavaillé (www.ehess.fr).

Voir la collection «Libertinage et philosophie au XVII ${ }^{\mathrm{e}}$ siècle», PUF Saint Etienne 2002 et suite, dirigée par A. McKenNA, où de nombreux articles réfléchissent sur la définition du libertinage. 
pour lui la foi, légitimée par une «époché», non de principe comme chez les pyrrhoniens, mais articulée nécessairement à un fidéisme moderne, qui a instrumentalisé la raison en chemin, puis l'a écartée au contraire du fidéisme tel qu'il se définit habituellement, c'est à dire qui met en doute le pouvoir de la raison.

L'acceptation de la science et son instrumentalisation rhétorique serait donc pour Bayle la voie moderne d'accession à la vraie foi, en dépit de l'échec apparent de la théologie rationnelle. La foi persiste, en dépit de l'épuisement des doctrines de son analyse. La raison est utilisée et écartée pour prouver la foi.

Nous n'y voyons pas précisément l'échec du rationalisme religieux contrairement à ce que dit la critique actuelle, mais l'instrumentalisation d'un concept abouti (de la finitude de ce concept en fait) au profit de l'irrationalisme de la foi. Sinon la foi serait une superstition comme une autre... Ce que Bayle ne suggère à aucun moment. Bacon dans son essai Of atheism souligne à ce titre un intéressant paradoxe «it's true that a little philosophy inclineth mind's man to atheism, but depth in philosophy bringeth men's minds about religions $>^{34}$. Et Bayle le loue dans le DHC, disant qu'il est l'un des plus grands esprits de son siècle (vol. I, p. 417).

Nous noterons pour terminer que le DHC, ses notes et éclaircissements, relativisent la portée rationaliste des PD, non dans son objet (la raison), mais dans ses limites. Bayle s'en distancie avec le temps, mais ne renie pas son nécessaire épuisement comme une étape qui laisse sa place à la foi. «Bayle en arrive à définir le mérite de la foi comme proportionnel à l'effort nécessaire pour surmonter les objections philosophiques» dit McKenna, au colloque de Nimègue (1996) ${ }^{35}$.

Selon nous le sacrifice de la raison est bien la preuve de la foi de Bayle. Et l'acceptation du doute empirique, l'expression sceptique de son tâtonnement. L'examen des rééditions successives des Pensées diverses sur la comète, du vivant de l'auteur, après la publication des Principia de Newton, ne montre pas une intégration radicale des progrès des nouveaux savoirs dans la démonstration de Bayle. Il semble rester sur la première position des PD de 1683, comme si les preuves scientifiques apportées par le célèbre physicien sur les comètes, n'ont qu'une importance relative dans sa dissertation ${ }^{36}$. Le but supérieur est bien l'expression du sentiment religieux dans ses nouveaux rapports avec la raison, à l'aube de la modernité.

ALEXANDRA WILLAUME ALBERTINI

(34) F. BACOn, The Essays or Councils, Civil and Moral, Essay XVI, Of atheism, with annotations by R. Whately, D.D., J.W. Parker and son, West Strand, London, 1858, p. 156. «un peu de philosophie incline les esprits vers l'athéisme, mais la profondeur philosophique les ramène à la religion».

(35) Voir aussi le collectif Pierre Bayle et la liberté de conscience, sous la direction de P. FRÉCHET, Ed. Anarchasis, Janvier 2012. Actes du colloque de
Carla-Bayle, Pierre Bayle héritier et médiateur de la liberté de conscience à l'âge classique, 2009.

(36) J'en propose la démonstration dans ma conférence au congrès de littérature comparée (AILC) de juillet 2013 à Paris. Savoir astronomique et postulations philosophiques dans la thèse de Bayle sur la comète, en comparant les progrès des savoirs en astro-physique aux postulations de Bayle au fil des rééditions des $\mathrm{PD}$. 